$$
\text { "papp" — 2008/12/19 — 14:45 — page } 101-\# 1
$$

\title{
GeoGebra in mathematics teaching
}

\author{
ZsuZsanna PAPP-VARga
}

Abstract. GeoGebra is a dynamic mathematics software which combines dynamic geometry and computer algebra systems into an easy-to-use package. Its marvel lies in the fact that it offers both the geometrical and algebraic representation of each mathematical object (points, lines etc.). The present article gives a sample of the potential uses of GeoGebra for mathematics teaching in secondary schools.

Key words and phrases: dynamic mathematics software, dynamic geometry system, computer algebra system.

ZDM Subject Classification: D40, N80, U70.

\section{An overview of mathematics softwares}

This section reviews the points that teachers may find worth considering while deciding on which type of software they choose to use for their mathematics teaching in teaching mathematics.

There are specific software packages (relating to fewer and smaller areas) and there are applications with a more general objective (that can be applied efficiently in several areas). For instance the program Graph dis suitable for teaching of functions while Maple touches upon almost all fields of mathematics. In addition, there are applications used worldwide while others are used only in particular countries. Some of the international applications have been translated into several languages, however, there are some that are available in one or the other world languages. Euklidesz for example was developed in Hungarian, but Cabri is also available in Hungarian. Different kinds of software may also vary according to

Copyright (c) 2008 by University of Debrecen 
what command of informatics is needed to use them. Some programmes such as Derive require only basic knowledge of the user while others need programming skills (e.g. Maple). Certain types of software are platform-independent while others operate only on certain platforms. Euklidesz for example runs well only on Windows whereas GeoGebra can be easily run on both Linux and Mac OS. Some types of software need to be installed while in other cases you only need to run the application. (The former types include for example Maple, however, GeoGebra Web start belongs to the latter group.) Certain software types can be freely used for teaching objectives, however, in other cases only a certain version of the software is available free of charge and there are some which you have to buy. Graph for that matter is completely free, while in the case of Euklidesz only its version 1.1 is available at no charge and as far as Maple is concerned, it exists only in commercial versions.

In order to save precious teaching time, it is worthwhile selecting a type of software that is applicable in various fields, thus you only need to allocate time to become familiar with the software only once. Of course, then you have to consider the potential difficulties the user might face due to the complexity of a multifunctional programme. In education, it is probably more expedient to choose a type of software that is available in the students' native language, thus eliminating potential difficulties arising from language problems (except cases when the teaching objectives include the grounding of the foreign (professional) language as well). It is generally more practical to select a type of software for teaching that does not require a higher knowledge of informatics unless the teaching and development of advanced informatics knowledge and skills are also among your priorities. For practical considerations, it is advantageous if the programme can operate on several platforms and does not require being installed. In public education the free availability of the programme is also a crucial factor since it offers both a cost-effective solution for the school budget and the possibility for students for further practice at home.

The mathematical software, GeoGebra, that will be introduced in the following chapters meets all the above expectations so it is worthy to call attention of each teacher of mathematics working in secondary education. 


$$
\text { "papp" — 2008/12/19 — 14:45 — page } 103 \text { — \#3 }
$$

\section{A brief introduction to GeoGebra}

Geogebra is a free, platform-independent dynamic mathematics software available in 40 languages. The topics can be taught with GeoGebra range from geometry, algebra to function analysis.

Its development started in 2001-2002 by Markus Hohenwarter as a part of his master's thesis at Salzburg University with the aim of providing additional educational aid for secondary schools. In his doctoral studies on mathematics education he carried out further developments on the programme. GeoGebra has won several international awards in the past few years and has been translated into 40 languages.

On the one hand, GeoGebra is a dynamic construction system. In fact, the user is provided with a virtual construction kit with the help of which they can prepare any kind of construction required in secondary education. In contrast to constructions carried out on paper, here the initial objects (points, straight lines etc.) are freely movable in such a way that the dependent objects move together.

On the other hand, it is a computer algebra system in which objects can be entered in an algebraic way (points together with their coordinates, straight lines together with their equations, functions together with their formulas etc.). It may also be used for calculations such as derivation and integration of functions etc.

One of the most important characteristic of GeoGebra, however, is probably its feature that an expression in the algebra window corresponds to an object in the geometry window and vice versa. Regardless of the way the object was entered (either by construction in the geometry window or by giving its parameters in the algebra window) it may be modified in either windows and the subsequent changes appear in the other window as well.

GeoGebra is a well-documented programme, and in addition to the free availability of the software in several languages, users can also easily access Help and the website of the software where additional information are available. Furthermore, readers may browse through GeoGebra-related publications uploaded on the website. 


$$
\text { "papp" — 2008/12/19 — 14:45 — page } 104 \text { — \#4 }
$$

The use of GeoGebra in teaching mathematics

About the use of mathematics softwares in general

This section covers the various potential uses of GeoGebra including the fields where it offers extra help to both teachers and students.

One of the key tasks in education is to motivate students and make them feel interested. Naturally, capable teachers had been able to motivate children even before computers became widespread without feeling the need for various types of software. Then the reader has every reason to ask why we should begin using software now? Well, first of all students have changed a lot since the world they live in has also undergone great changes. Secondly, the more varied tools teachers have, the more efficient they become. Last but not least, mathematics software very often provides teachers with such new tools that are not replaceable with anything else.

Besides motivation, another major task is differentiation, since in almost every case teachers have to deal with heterogeneous groups. Using software, teachers are able to create extra materials of various content and level thus becoming able to properly differentiate between students.

A substantial part of teachers' work is the preparation of different types of extra material such as test banks, progress tests, summaries etc. The use of various software utilities facilitate and accelerate this task. With the help of GeoGebra teachers are able to easily create precise and spectacular figures. Moreover, they can publish their dynamic worksheets on the Internet at ease, making them accessible for their students.

\section{Introducing the potential application of GeoGebra}

In the following section it will be introduced how to use GeoGebra at the various stages of the teaching process by discussing the topic of a circle drawn around a triangle.

Before introducing new topics in mathematics lessons it is often inevitable to review previous skills and knowledge. The ability to construct the perpendicular bisectors of a triangle is a prerequisite for constructing a circumscribed circle. The necessary steps might be demonstrated by the teacher through an animation or students might be asked to draw it on their own with the help of the programme. The result will be certainly faster and more precise compared to constructions carried out manually. 
In the course of teaching new topics, the revision is followed by raising a new question, highlighting the problem. In the given topic the teacher may ask the following questions: 'What do you establish about the points of intersection of the perpendicular bisectors?', 'Where do you find the common point of intersection compared to the triangle?' In both cases, the manually created, static figures help students much less than a dynamic figure where you can easily change the triangle into an acute angled, right-angled or obtuse-angled one by moving its vertices.

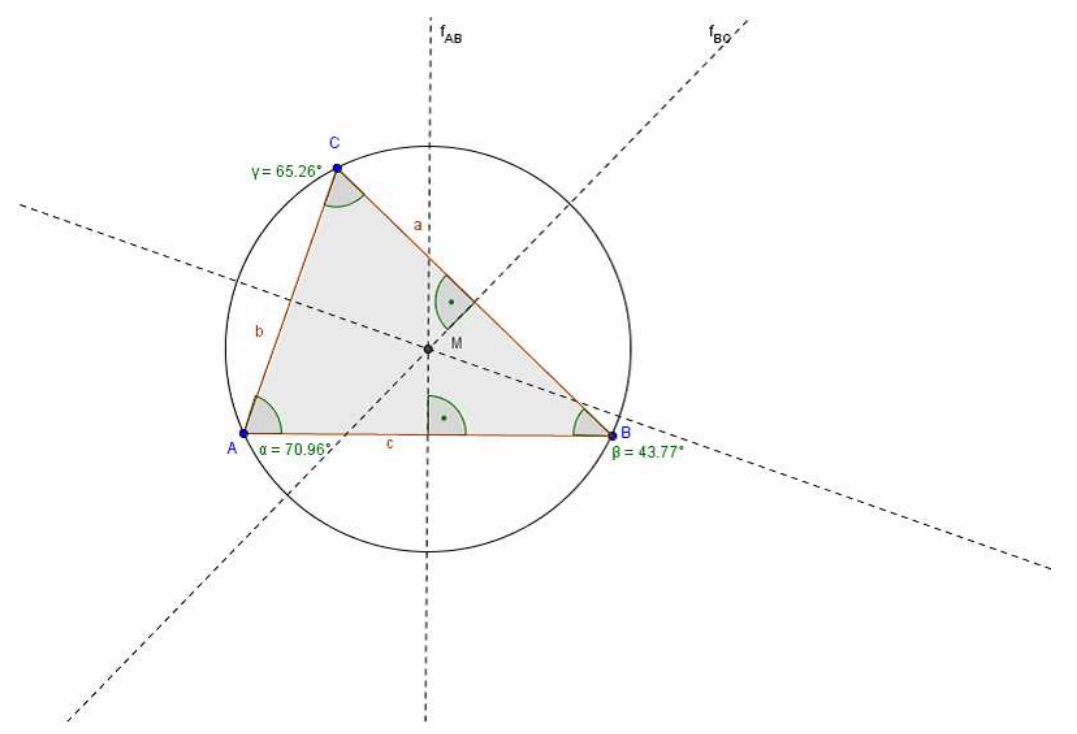

Figure 1. Circumscribed circle of a triangle

Once students have the conjecture they can start the proof. A suitable figure or animation might be of great help to explain proof. In our case you have the possibility to prepare an animation presenting the proof step by step while you can also put forward the question relating to the length of $A M, B M$ and $C M$ sections which may assist students to deduce the theorem.

The proof of theorems might be followed by exercises requiring the application of what we have just verified. With the help of GeoGebra software both teachers and the students can easily introduce, solve and check these exercises. As a result of the dynamism of the programme, it is easy to examine special cases (rendering an easy discussion possible) while the features of standard problems 


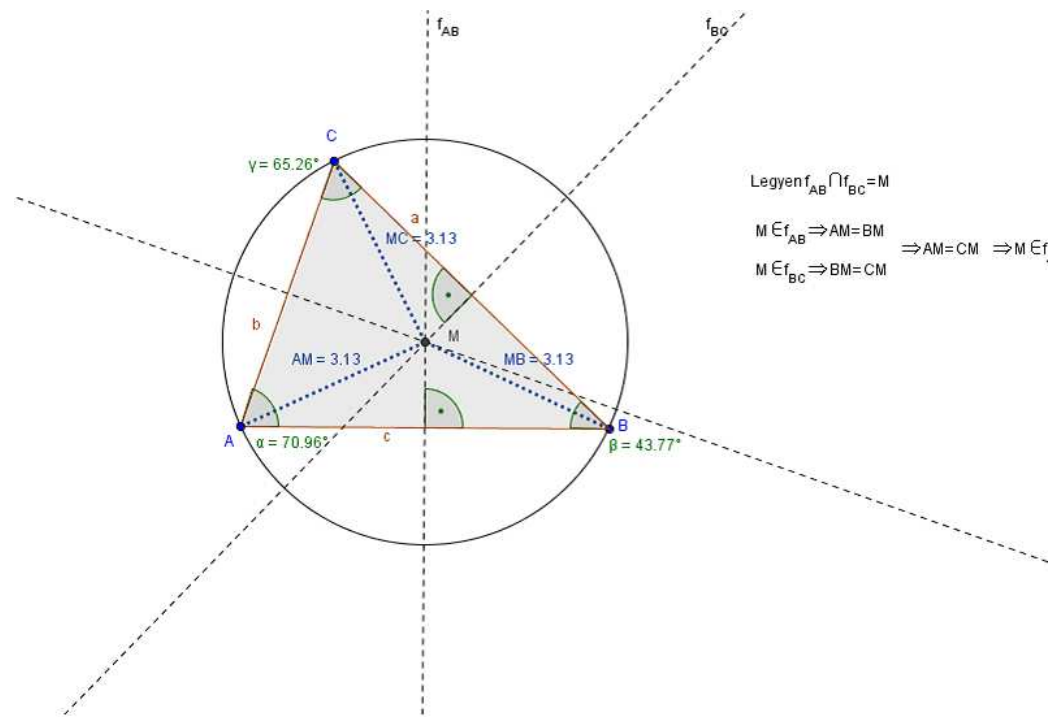

Figure 2. The verification of the theorem

become completely exposed. In the given topic for example you might give students the following task: Use GeoGebra to construct the circumscribed circle of $A B C$ triangle if $A B=4 \mathrm{~cm}, \alpha=60^{\circ}$ and $\beta=45^{\circ}$ !

Once the exercise is solved, with the help of the software you can easily examine how the figure changes in case different parameters are given: e.g. what happens if $\alpha=30^{\circ}$ and $\beta=60^{\circ}$ ?

GeoGebra can be also helpful while giving summaries. The software offers teachers with an opportunity to give a demonstration by highlighting only the crucial parts and correlations. In our case, for example, at the end of the class you do not need to discuss the construction steps of the perpendicular bisectors again, it is sufficient to direct attention only to Figure 2 with the help of which you can revise the material of the whole lesson. When one wishes to revise standard problems while summarizing comprehensive area, the software offers great help again since you have nothing else to do but show an application that provides animations for each standard problem. Finally, the software can also fosters students' learning due to the fact that they can easily revise any particular topic by referring back to all the extra materials they prepared during previous classes. 


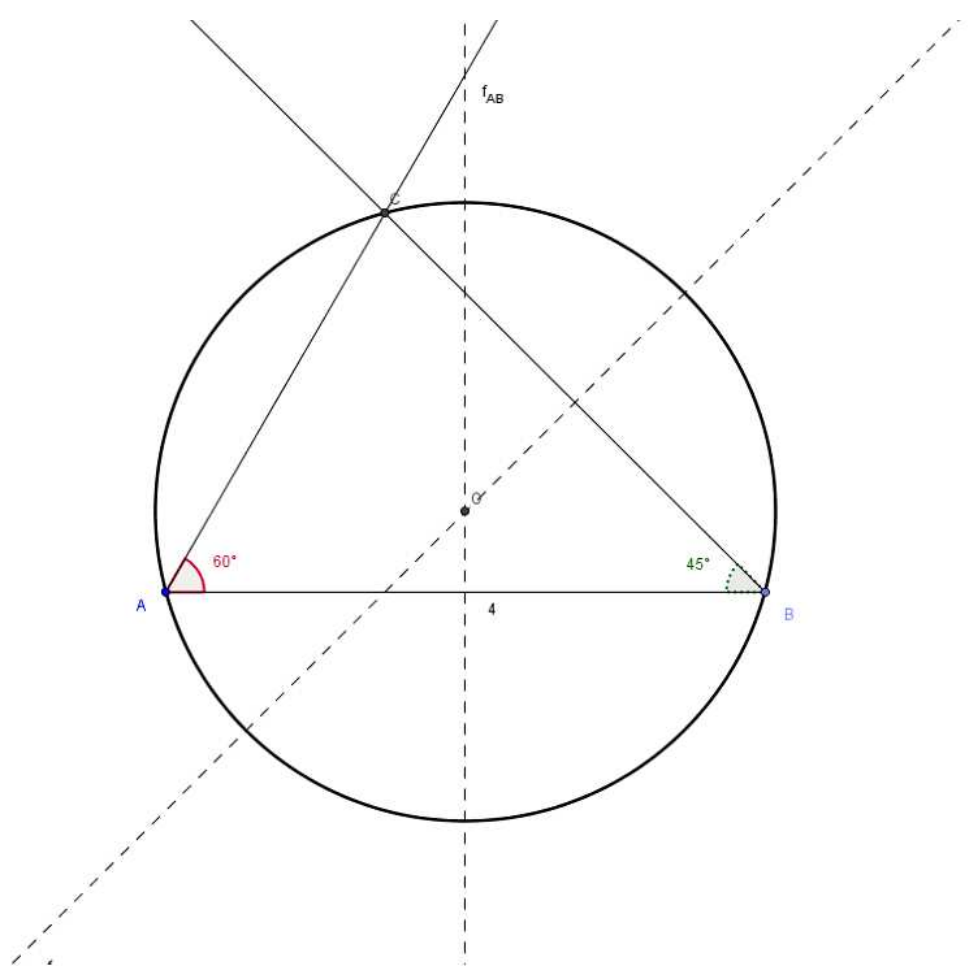

Figure 3. Solution of the given exercise

\section{Integrating GeoGebra into teaching practice}

One of the most crucial prerequisite of integrating GeoGebra into the teaching practice is perhaps the beliefs and additudes of teachers. In many cases teachers become interested in trying new tools if lectures and demonstration lessons convince them that these tools are worth using. If a teacher intends to incorporate GeoGebra or any other mathematics software into their everyday practice then the first step is to get thoroughly acquainted with the programme. One of the priorities of GeoGebra institutions that are being established all over the world will be to co-ordinate the two tasks mentioned above i.e. to organize lectures, demonstration lessons and workshops. Another common obstacle to the integration of softwares into daily routine is the teacher's lack of courage and time to construct their own teaching materials. From the GeoGebra wiki teachers can 


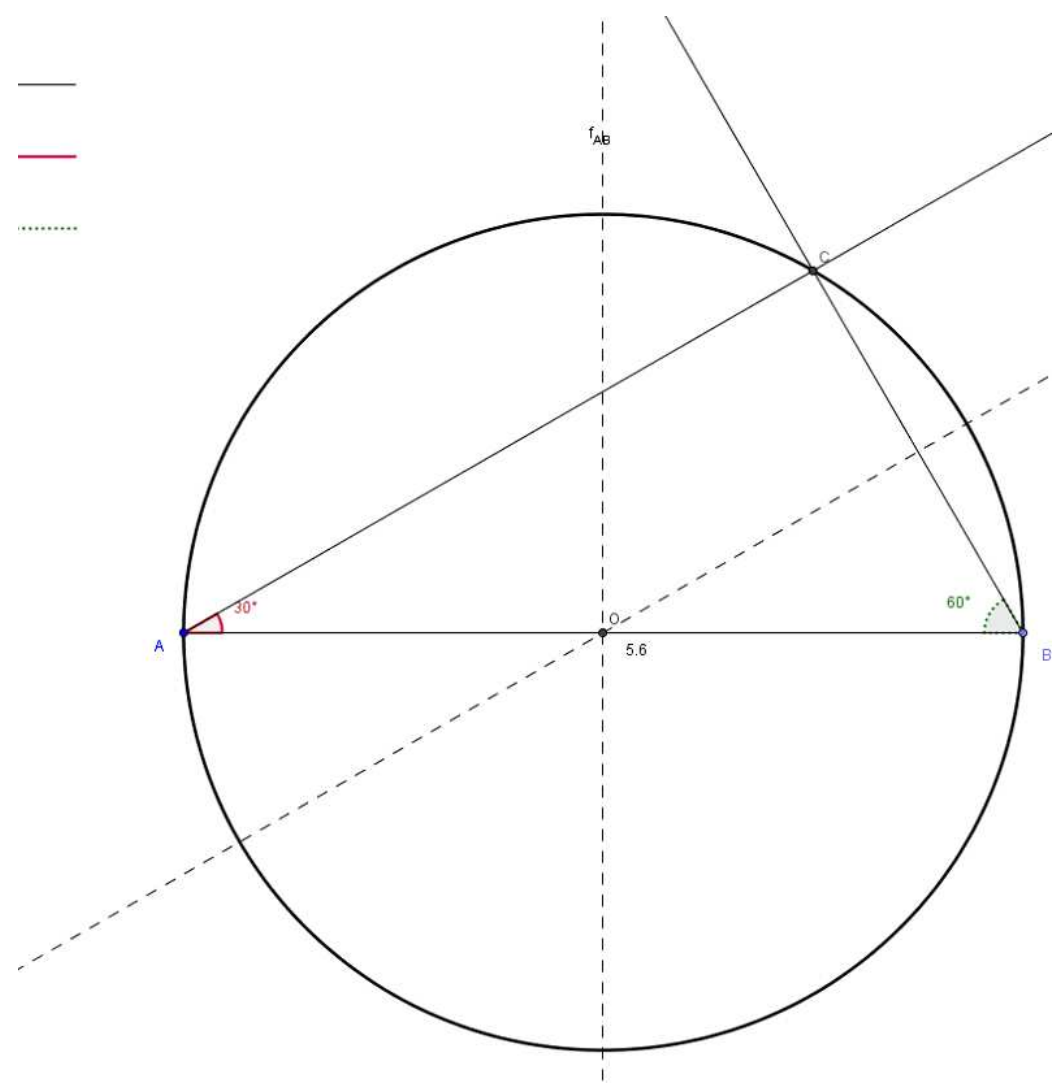

Figure 4. Examining the standard problem

easily download their colleagues' material which then they can tailor to their own needs.

Besides personal disposition, certain pieces of equipment should also be provided. Today most schools equipped with computers with projectors or smart boards in each class. In many schools there are several computers installed, and sometimes each student has access to their own computer. Thus, facilities and the set teaching objectives together determine how teachers organize their teaching process supported by GeoGebra: they can use a teacher-centred method or students may work alone, in pairs or small groups.

In this paper, readers could learned about applications of GeoGebra in mathematics classrooms. The software provides excellent opportunities for extra classes 


$$
\text { "papp" — 2008/12/19 — 14:45 — page } 109 \text { — \#9 }
$$

or study groups as well to examine more complex or advanced problems such as derivation, integration, transformation, or matrixes just to mention a few. Working with this software develops not only mathematical but IT skills as well, a preferred solution could be to teach students how to use the software in IT classes and then later they can use that knowledge during mathematics classes. It is also important to note the role of GeoGebra in individual learning. With the help of the extra materials students can revise what they have learnt in class and also do or check their homework at home. In addition, those with an entrepreneurial spirit may start searching for new correlations and making new discoveries in the world of mathematics.

\section{Conclusion}

I hope that the the examples offered considerations for readers as to why it is worthwhile using mathematics utility softwares and what considerations one should bear in mind when choosing a particular type of software. I conclude my remarks in the hope that by briefly demonstrating how to apply GeoGebra on a given topic you will feel inclined to use it in your everyday practice.

In addition to 'empty' worksheets, it is of crucial importance that in the future teachers also have access to methodological support materials that provide excellent and easy-to-use aid in everyday practice, just like the one described in the present paper. In order to achieve this objective it is desirable to develop, test and optimize as many similar materials as possible.

\section{References}

[1] Markus Hohenwarter, GeoGebra 2.5 Handbook, 2006, translated into Hungarian by Szabolcs Sulik, http://www.geogebra.org/help/docuhu/.

[2] Markus Hohenwarter, Judith Preiner, Dynamic Mathematics with GeoGebra, 2007, http://www.maa.org/joma/Volume7/Hohenwarter/index.html.

[3] Dr. Karl Josef Fuchs, Computer Algebra Systems in Mathematics Teaching - Teacher Training Programmes, Challenges and New Objectives, 2002, http://matserv.pmmf.hu/anniv/cd_hun/prezentaciok/fuchs.pdf.

[4] Tamás Árki, Teaching Feuerbach's Circle with the Help of computers, http://www.sulinet.hu/tart/fcikk/Kcn/0/22581.

[5] István László, Péter Simon, Euklides: the Dynamic Geometry Construction Programme, 2002, http://matserv.pmmf.hu/anniv/cd_hun/prezentaciok/laszlosimon.pdf. 
$\bigoplus$

$$
\text { "papp" — 2008/12/19 - 14:45 — page } 110-\# 10
$$

[6] Iván Falusi, Didactics (Theoretical Basis for Learning Teaching), National Textbook Publisher, Budapest.

ZSUZSANNA PAPP-VARGA

ELTE FACULTY OF INFORMATICS

BUDAPEST

HUNGARY

E-mail: vzsuzsa@elte.hu

(Received June, 2008) 